\title{
Tau Neutrino Astronomy in GeV Energies
}

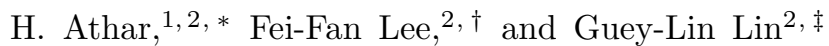 \\ ${ }^{1}$ Physics Division, National Center for Theoretical Sciences, Hsinchu 300, Taiwan \\ ${ }^{2}$ Institute of Physics, National Chiao-Tung University, Hsinchu 300, Taiwan
}

(Dated: November 19, 2018)

\begin{abstract}
We point out the opportunity of the tau neutrino astronomy for the neutrino energy $E$ ranging between $10 \mathrm{GeV}$ and $10^{3} \mathrm{GeV}$. In this energy range, the intrinsic tau neutrino production is suppressed relative to the intrinsic muon neutrino production. Any sizable tau neutrino flux may thus arise because of the $\nu_{\mu} \rightarrow \nu_{\tau}$ neutrino oscillations only. It is demonstrated that, in the presence of the neutrino oscillations, consideration of the neutrino flavor dependence in the background atmospheric neutrino flux leads to the drastically different prospects between the observation of the astrophysical muon neutrinos and that of the astrophysical tau neutrinos. Taking the galactic-plane neutrino flux as the targeted astrophysical source, we have found that the galactic-plane tau neutrino flux dominates over the atmospheric tau neutrino flux for $E \geq 10 \mathrm{GeV}$. Hence, the galactic-plane can at least in principle be seen through the tau neutrinos with energies just greater than $10 \mathrm{GeV}$. In a sharp contrast, the galactic-plane muon neutrino flux is overwhelmed by its atmospheric background until $E \geq 10^{6} \mathrm{GeV}$.

PACS numbers: 98.38.-j, 13.85.Tp, 14.60.Pq
\end{abstract}

\section{INTRODUCTION}

The $\nu_{\mu} \rightarrow \nu_{\tau}$ neutrino oscillations established by the high-statistics Super-Kamiokande (SK) detector, ensure that a non-negligible $\nu_{\tau}$ flux reaches the Earth. A recent SK analysis of the atmospheric neutrino data implies the following range of the neutrino mixing parameters [1]

$$
\delta m^{2}=(1.9-3.0) \cdot 10^{-3} \mathrm{eV}^{2}, \sin ^{2} 2 \theta>0.9 .
$$

This is a $90 \%$ C.L. range of the neutrino mixing parameters with the best fit values given by $\delta m^{2}=2.4 \cdot 10^{-3} \mathrm{eV}^{2}$ and $\sin ^{2} 2 \theta=1$ respectively.

The tau neutrinos resulting from the above $\nu_{\mu} \rightarrow \nu_{\tau}$ oscillations are presently identified on the statistical basis 2]. On the other hand, the total number of observed nontau neutrinos from various detectors are already greater than $\sim 10^{4}$ with energies ranging from $\sim 10^{-1} \mathrm{GeV}$ to $\sim$ $10^{3} \mathrm{GeV}[3]$. It is essential to develop efficient techniques to identify the tau neutrinos [4].

There are at least two important reasons for observing the $\nu_{\tau}$. First, seeing the $\nu_{\tau}$ confirms the $\nu_{\mu} \rightarrow \nu_{\tau}$ oscillation interpretation for the atmospheric neutrino data. Second, since the atmospheric $\nu_{\tau}$ flux is generally suppressed as compared to the atmospheric $\nu_{\mu}$ flux, the prospective observation of the astrophysical $\nu_{\tau}$ suffers much less background than in the $\nu_{\mu}$ case.

In this paper, we address the second point with the galactic-plane tau neutrinos as our illustrating astrophysical source. We point out that contrary to the general expectations, the atmospheric neutrino flux does not

\footnotetext{
*Electronic address: athar@phys.cts.nthu.edu.tw

${ }^{\dagger}$ Electronic address: u1717117.py87g@nctu.edu.tw

‡Electronic address: glin@cc.nctu.edu.tw
}

dominate over the astrophysical neutrino flux for the neutrino energy $E<10^{3} \mathrm{GeV}$, once the flavor composition of the neutrino flux is taken into account. The idea for such an investigation has appeared earlier in Ref. [5].

In the context of two neutrino flavors, $\nu_{\mu}$ and $\nu_{\tau}$, the total tau neutrino flux arriving at the detector on Earth, after traversing a distance $L$, is

$$
\phi_{\nu_{\tau}}^{\text {tot }}(E)=P(E) \cdot \phi_{\nu_{\mu}}(E)+(1-P(E)) \cdot \phi_{\nu_{\tau}}(E),
$$

where $P(E) \equiv P\left(\nu_{\mu} \rightarrow \nu_{\tau}\right)=\sin ^{2} 2 \theta \cdot \sin ^{2}\left(L / L_{\mathrm{osc}}\right)$ with the neutrino oscillation length given by $L_{\mathrm{osc}}=4 E / \delta \mathrm{m}^{2}$.

In order to compute the total $\nu_{\tau}$ flux from a given astrophysical site, we need to first compute the intrinsic $\nu_{\mu}$ as well as the intrinsic $\nu_{\tau}$ flux from the same site.

\section{GALACTIC-PLANE NEUTRINO FLUX}

One calculates the intrinsic galactic-plane $\nu_{\mu}$ and $\nu_{\tau}$ fluxes by considering the collisions of incident cosmicray protons with the interstellar medium. The fluxes are given by

$$
\phi_{\nu}(E)=R n_{p} \int_{E}^{\infty} \mathrm{d} E_{p} \phi_{p}\left(E_{p}\right) \frac{\mathrm{d} \sigma_{p p \rightarrow \nu+Y}}{\mathrm{~d} E},
$$

where $E_{p}$ is the energy of the incident cosmic-ray proton, $\mathrm{d} \sigma_{p p \rightarrow \nu+Y} / \mathrm{d} E$ is the $\nu$ energy spectrum in the $p p$ collisions. $R$ is the typical distance in the galaxy along the galactic-plane, which we take as $10 \mathrm{kpc}\left(1 \mathrm{pc} \sim 3 \cdot 10^{13}\right.$ $\mathrm{km})$. The density of the interstellar medium $n_{p}$ along the galactic plane is taken to be $\sim 1$ proton per $\mathrm{cm}^{3}$. The primary cosmic-ray proton flux, $\phi_{p}\left(E_{p}\right) \equiv \mathrm{d} N_{p} / \mathrm{d} E_{p}$, is given by []

$$
\phi_{p}\left(E_{p}\right)=1.49 \cdot\left(E_{p}+2.15 \cdot \exp \left(-0.21 \sqrt{E_{p}}\right)\right)^{-2.74},
$$






FIG. 1: The intrinsic galactic-plane and the intrinsic downward going atmospheric muon and tau neutrino fluxes as a function of the neutrino energy in $\mathrm{GeV}$. Details are provided in the text.

in units of $\mathrm{cm}^{-2} \mathrm{~s}^{-1} \mathrm{sr}^{-1} \mathrm{GeV}^{-1}$. The above flux is under the assumption that the cosmic-ray flux spectrum in the galaxy is a constant and equal to its locally observed value. The galactic-plane neutrino flux (abbreviated here as Gal) is sometimes also referred to as the galactic center region neutrino flux (abbreviated as G or GC), the galactic disk neutrino flux or the Milky Way neutrino flux. We shall estimate here the neutrino flux coming from the galactic-plane direction only as transverse to it, the $n_{p}$ decreases essentially exponentially [7], and so does the $\phi_{\nu}(E)$ according to Eq. (3).

The neutrino production process $p+p \rightarrow \nu+Y$ is mediated by the production and the decays of the $\pi$, the $K$, and the charmed hadrons. The galactic muon neutrinos mainly come from the two-body $\pi$ decays and the subsequent three-body muon decays. While the decay rates and the decay distributions of the $\pi$ and the $\mu$ are well understood, the differential cross section for the process $p+p \rightarrow \pi+Y$ is model dependent. We adopt the parameterization for this cross section from Ref. [8], which is obtained by using the accelerator data in the sub- $\mathrm{TeV}$ energy range [9]. We remark that our galactic-plane $\nu_{\mu}$ flux compares well with a previous calculation [7] using the PYTHIA [10].

The galactic-plane $\nu_{\tau}$ flux arises from the production and the decays of the $D_{s}$ mesons. It has been found to be rather suppressed compared to the corresponding $\nu_{\mu}$ flux [1]. Fig. [1 shows the intrinsic galactic-plane $\nu_{\mu}$ and $\nu_{\tau}$ fluxes obtained by using the above description (along with the corresponding intrinsic downward going atmospheric neutrino fluxes). We shall use these in our subsequent estimates.

The total galactic-plane tau neutrino flux, $\phi_{\nu_{\tau}}^{\text {tot }}(E)$, is therefore dominated by the $\nu_{\mu} \rightarrow \nu_{\tau}$ oscillations indicated by the term $P(E) \cdot \phi_{\nu_{\mu}}(E)$ in Eq. (2). With the best-fit values for the neutrino mixing parameters, we have $\phi_{\nu_{\tau}}^{\text {tot }}(E) \approx \phi_{\nu_{\mu}}(E) / 2$, neglecting the contribution of $\phi_{\nu_{\tau}}(E)$, since $L_{\mathrm{osc}} \ll L$, where $L \sim 5 \mathrm{kpc}$. The total galactic-plane tau neutrino flux, $\phi_{\nu_{\tau}}^{\text {tot }}(E) \equiv$ $\mathrm{d} N / \mathrm{d}\left(\log _{10} E\right)$, can be parameterized for $1 \mathrm{GeV}^{\tau} \leq E \leq$ $10^{3} \mathrm{GeV}$, as

$$
\phi_{\nu_{\tau}}^{\mathrm{tot}}(E)=A\left(\frac{E}{\mathrm{GeV}}\right)^{\alpha}
$$

where $A=2 \cdot 10^{-5}$ is in units of $\mathrm{cm}^{-2} \mathrm{~s}^{-1} \mathrm{sr}^{-1}$ with $\alpha=$ -1.64 .

\section{ATMOSPHERIC NEUTRINO FLUX}

The calculation of the total atmospheric tau neutrino flux is more involved. We follow the approach in Ref. [8] for computing the intrinsic atmospheric $\nu_{\mu}$ flux which can oscillate into $\nu_{\tau}$.

For the $\pi$-decay contribution, the flux formula reads:

$$
\begin{aligned}
\frac{\mathrm{d}^{2} N_{\nu_{\mu}}^{\pi}(E, \xi, X)}{\mathrm{d} E \mathrm{~d} X}= & \int_{E}^{\infty} \mathrm{d} E_{N} \int_{E}^{E_{N}} \mathrm{~d} E_{\pi} \frac{\Theta\left(E_{\pi}-\frac{E}{1-\gamma_{\pi}}\right)}{d_{\pi} E_{\pi}\left(1-\gamma_{\pi}\right)} \\
& \times \int_{0}^{X} \frac{\mathrm{d} X^{\prime}}{\lambda_{N}} P_{\pi}\left(E_{\pi}, X, X^{\prime}\right) \\
& \times \frac{1}{E_{\pi}} F_{N \pi}\left(E_{\pi}, E_{N}\right) \\
& \times \exp \left(-\frac{X^{\prime}}{\Lambda_{N}}\right) \phi_{N}\left(E_{N}\right)
\end{aligned}
$$

where $E$ is the neutrino energy and $\xi$ is the zenith angle in the direction of the incident cosmic-ray nucleons. The $\gamma_{\pi}=m_{\mu}^{2} / m_{\pi}^{2}$ and $d_{\pi}$ is the pion decay length in units of $\mathrm{g} / \mathrm{cm}^{2}$. The $\lambda_{N}$ is the nucleon interaction length while $\Lambda_{N}$ is the corresponding nucleon attenuation length. $\phi_{N}\left(E_{N}\right)$ is the primary cosmic-ray spectrum. We only consider the proton component of $\phi_{N}$, which is given by Eq. (4). We take $\lambda_{N}=86 \mathrm{~g} / \mathrm{cm}^{2}$ and $\Lambda_{N}=120 \mathrm{~g} / \mathrm{cm}^{2}$ [9]. The function $P_{\pi}\left(E_{\pi}, X, X^{\prime}\right)$ is the probability that a charged pion produced at the slant depth $X^{\prime}\left(\mathrm{g} / \mathrm{cm}^{2}\right)$ survives to the slant depth $X\left(>X^{\prime}\right)$. The $F_{N \pi}\left(E_{\pi}, E_{N}\right)$ is the normalized inclusive cross section for $N+$ air $\rightarrow \pi^{ \pm}+Y$, and is given in the Ref. [8].

The kaon contribution to the atmospheric $\nu_{\mu}$ flux has the same form as Eq. (6) with an inclusion of the branching ratio $B(K \rightarrow \mu \nu)=0.635$ and appropriate replacements in the kinematics factors as well as in the normalized inclusive cross section. We remark that the current approach neglects the 3-body muon-decay contribution to the $\nu_{\mu}$ flux. This is a good approximation for $E>10$ 


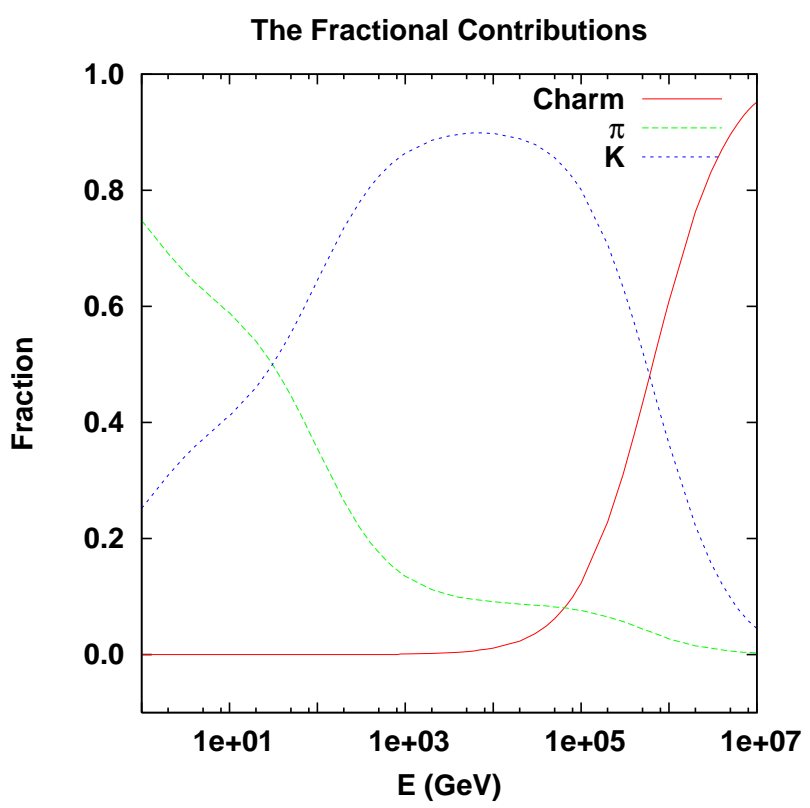

FIG. 2: The fraction of contributions by the $\pi$, the $K$, and the charm decays to the overall downward going atmospheric $\nu_{\mu}$ flux as a function of the neutrino energy in $\mathrm{GeV}$.

$\mathrm{GeV}$ [8]. The relevance of the muon-decay contribution for $1 \mathrm{GeV} \leq E \leq 10 \mathrm{GeV}$ will be commented later.

We stress that the $\pi$ and the $K$ decays are not the only sources for the atmospheric $\nu_{\mu}$ flux. For $E>10^{6} \mathrm{GeV}$, the charm-decay contribution becomes more important than those of the $\pi$ and the $K$ decays. We have used the results from the perturbative QCD to estimate this contribution 12. The muon neutrino flux due to charm contribution can be written as

$$
\frac{\mathrm{d}^{2} N_{\nu_{\mu}}^{c}(E, X)}{\mathrm{d} E \mathrm{~d} X}=\sum_{h} \frac{Z_{p h} Z_{h \nu_{\mu}}}{1-Z_{p p}(E)} \cdot \frac{\exp \left(-X / \Lambda_{p}\right) \phi_{p}(E)}{\Lambda_{p}},
$$

where $h$ stands for the $D^{ \pm}$, the $D^{0}$, the $D_{s}$ and the $\Lambda_{c}$ hadrons. The $Z$ moments on the RHS of the equation are defined by

$$
Z_{i j}\left(E_{j}\right) \equiv \int_{E_{j}}^{\infty} \mathrm{d} E_{i} \frac{\phi_{i}\left(E_{i}\right)}{\phi_{i}\left(E_{j}\right)} \frac{\lambda_{i}\left(E_{j}\right)}{\lambda_{i}\left(E_{i}\right)} \frac{\mathrm{d} n_{i A \rightarrow j Y}\left(E_{i}, E_{j}\right)}{\mathrm{d} E_{j}},
$$

with $\mathrm{d} n_{i A \rightarrow j Y}\left(E_{i}, E_{j}\right) \equiv \mathrm{d} \sigma_{i A \rightarrow j Y}\left(E_{i}, E_{j}\right) / \sigma_{i A}\left(E_{i}\right)$. In the decay process, the scattering length $\lambda_{i}$ is replaced by the decay length $d_{i}$, whereas the $\mathrm{d} n_{i A \rightarrow j Y}\left(E_{i}, E_{j}\right)$ is replaced by the $\mathrm{d} \Gamma_{i \rightarrow j Y}\left(E_{i}, E_{j}\right) / \Gamma_{i}\left(E_{i}\right)$. We note that this part of the atmospheric $\nu_{\mu}$ flux is isotropic, unlike the contributions from the $\pi$ and the $K$ decays. This difference is attributable to the lifetime difference between the charm and the $\pi(K)$ mesons. The various $Z$ moments are calculated using the next-to-leading order perturbative QCD [13, 14]. The decay moments $Z_{h \nu_{\mu}}$ are calculated by using the charmed-hadron decay distributions given in Refs. 15, 16.

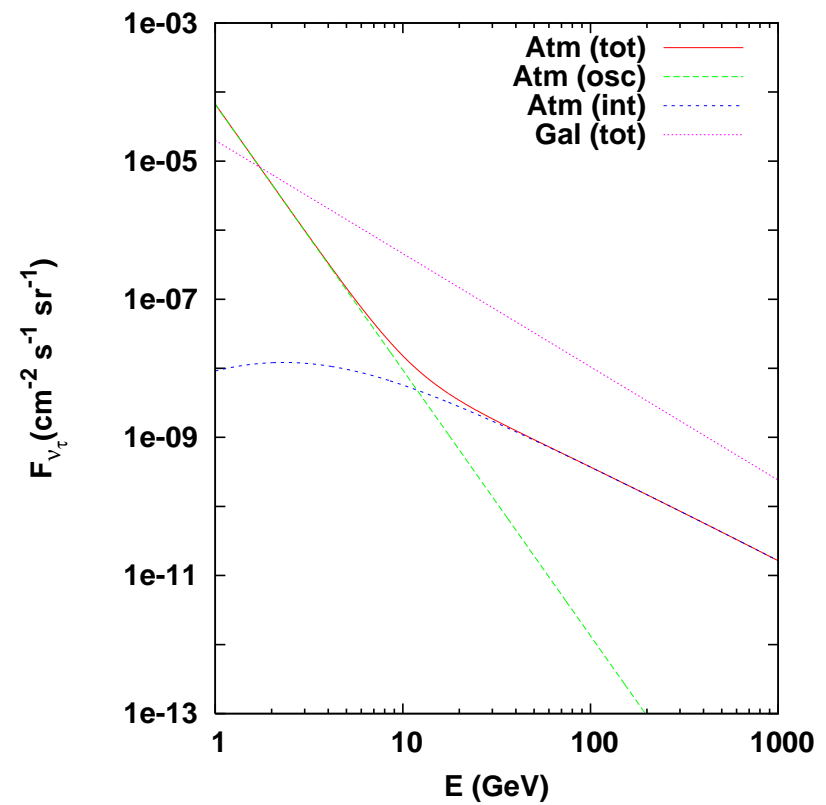

FIG. 3: Comparison of the total galactic plane tau neutrino flux with the total downward going atmospheric neutrino flux for the best fit values of the neutrino mixing parameters as a function of the neutrino energy. The intrinsic and oscillated parts of the total downward going atmospheric tau neutrino flux are also shown as a function of the neutrino energy.

We show the relative contributions by the $\pi$, the $K$, and the charm decays to the overall $\nu_{\mu}$ flux in Fig. 2] This is an extension of the Fig. 3 in Ref. [8], where only the $\pi$ and the $K$ contributions are compared. It is obvious that the $\pi$ decay contribution dominates for $1 \mathrm{GeV} \leq E \leq 10 \mathrm{GeV}$, while the $K$ decay contribution dominates between $10^{3} \mathrm{GeV}$ and $10^{5} \mathrm{GeV}$. The fraction of the charm-decay contribution rises rapidly at $E \geq 10^{5}$ $\mathrm{GeV}$ and becomes dominant for $E>10^{6} \mathrm{GeV}$. In this energy range, both the $\pi$ and the $K$ lose large fractions of their energies before decaying into the neutrinos.

Additionally, the intrinsic atmospheric $\nu_{\tau}$ flux is also required to completely determine the total atmospheric $\nu_{\tau}$ flux. This flux is calculated using the perturbative QCD, since $\tau$ neutrino arises from the $D_{s}$ decays [17]. The flux is written as Eq. (7) with $Z_{p h}$ replaced by the $Z_{p D_{s}}$ and $Z_{h \nu_{\tau}}$ replaced by the $Z_{D_{s} \nu_{\tau}}$. We note that the $Z_{D_{s} \nu_{\tau}}$ contains two contributions. One arises from the decay $D_{s} \rightarrow \nu_{\tau} \tau$; the other follows from the subsequent tau-lepton decay, $\tau \rightarrow \nu_{\tau}+Y$. The latter contribution is calculated using the decay distributions of the decay modes $\tau \rightarrow \nu_{\tau} \rho, \tau \rightarrow \nu_{\tau} \pi, \tau \rightarrow \nu_{\tau} a_{1}$ [17, 18], and the $\tau \rightarrow \nu_{\tau} l \nu_{l}$ [9, 16] (Fig. 1) shows the intrinsic downward going atmospheric muon and tau neutrino fluxes for 1 $\left.\mathrm{GeV} \leq E \leq 10^{3} \mathrm{GeV}\right)$

We have calculated the $\phi_{\nu_{\tau}}^{\text {tot }}(E)$ by applying Eq. (2) with $\phi_{\nu_{\mu, \tau}}(E)$ given by $\mathrm{d}^{2} N_{\nu_{\mu, \tau}}(E, X) / \mathrm{d} E \mathrm{~d} X$ and integrating over the slant depth $X$. For $\xi<70^{\circ}$, the oscillation probability $P\left(\nu_{\mu} \rightarrow \nu_{\tau}\right)$ is calculated using the 


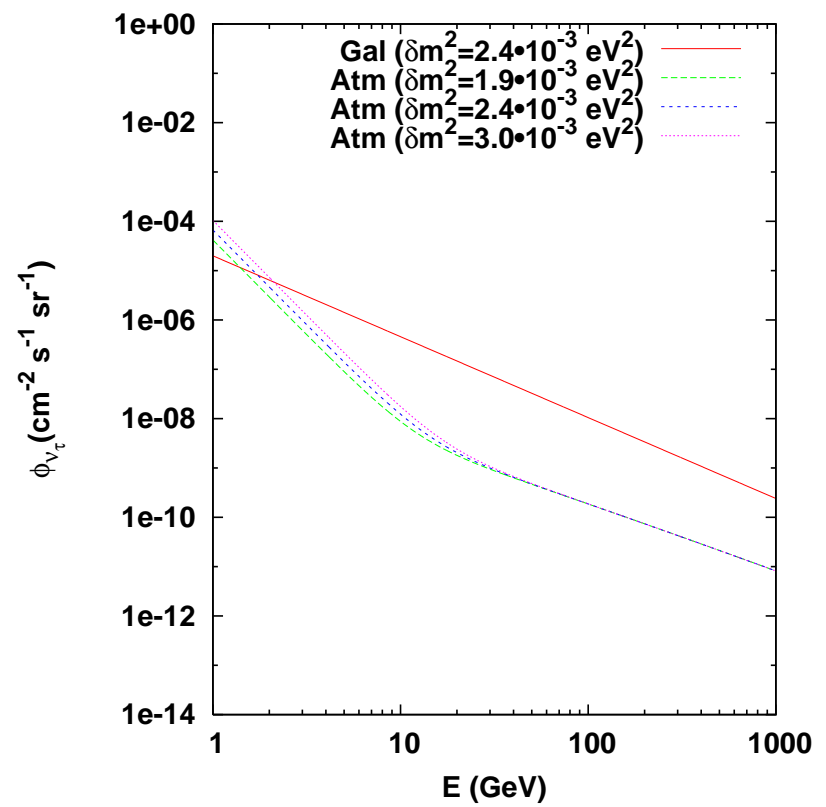

FIG. 4: The comparison of the galactic-plane and the downward going atmospheric $\nu_{\tau}$ fluxes in the presence of neutrino oscillations with maximal mixing as a function of the neutrino energy in $\mathrm{GeV}$. For $\delta m^{2}=2.4 \cdot 10^{-3} \mathrm{eV}^{2}$, both fluxes cross at $E=2.3 \mathrm{GeV}$.

relation $X=X_{0} \exp \left(-L \cos \xi / h_{0}\right) / \cos \xi$ with $X_{0}=1030$ $\mathrm{g} / \mathrm{cm}^{2}$ and $h_{0}=6.4 \mathrm{~km}$. Here $L$ is the linear distance from the neutrino production point to the detector on the Earth [19]. This gives, for instance, $P\left(\nu_{\mu} \rightarrow \nu_{\tau}\right) \simeq$ $\sin ^{2}\left(4.5 \cdot 10^{-2}(\mathrm{GeV} / E)\right)$ at $\xi=0^{\circ}$, for the best-fit values of the neutrino mixing parameters.

\section{THE COMPARISON}

The comparison of the galactic-plane and the downward going atmospheric $\nu_{\tau}$ flux is given in Fig. 3 and Fig. 4 in the presence of neutrino oscillations (we plot $\mathrm{d} N_{\nu} / \mathrm{d}\left(\log _{10} E\right)$ instead of $\left.\mathrm{d} N_{\nu} / \mathrm{d} E\right)$. The former flux clearly dominates the latter for $E \geq 10 \mathrm{GeV}$, whereas the two fluxes cross at $E=2.3 \mathrm{GeV}$ for $\delta m^{2}=2.4 \cdot 10^{-3}$ $\mathrm{eV}^{2}$ and $\sin ^{2} 2 \theta=1$. This comparison is however subject to the uncertainty of galactic-plane $\nu_{\tau}$ flux by the choices of the density $n_{p}$ and the distance $R$ mentioned before.

One can see that the atmospheric $\nu_{\tau}$ flux is sensitive to the value of $\delta m^{2}$ for $E \leq 20 \mathrm{GeV}$. Furthermore, a change of slope occurs for the atmospheric $\nu_{\tau}$ flux at $E \approx 20 \mathrm{GeV}$. Beyond this energy, the slope of the atmospheric $\nu_{\tau}$ flux is identical to that of the galactic-plane $\nu_{\tau}$ flux. For $E>20 \mathrm{GeV}$, the atmospheric $\nu_{\tau}$ flux is intrinsic, i.e., coming from the $D_{s}$ decays, whereas the galactic-plane $\nu_{\tau}$ flux arises from the oscillation of the $\nu_{\mu}$, which is produced mainly by the $\pi$ decays. In both cases, the hadrons decay before interacting with the medium. Such a feature dictates the slope of the outgoing neu-

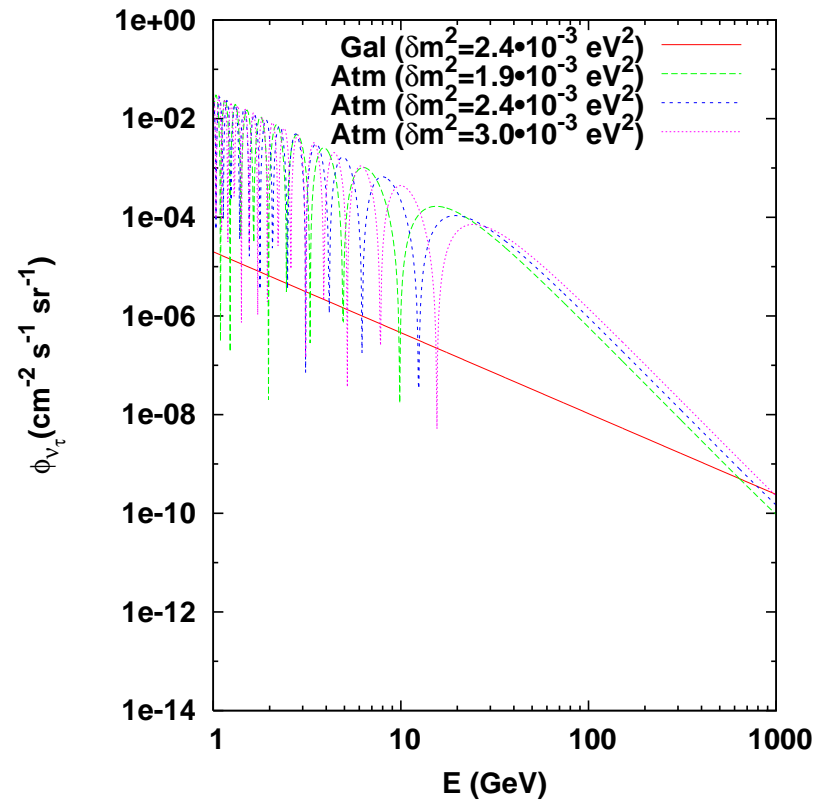

FIG. 5: The comparison of the galactic-plane $\nu_{\tau}$ flux and the atmospheric $\nu_{\tau}$ flux coming in the zenith angle $\xi=180^{\circ}$ in the presence of neutrino oscillations with maximal mixing as a function of the neutrino energy in $\mathrm{GeV}$. For $\delta m^{2}=2.4 \cdot 10^{-3}$ $\mathrm{eV}^{2}$, both fluxes cross at $E=800 \mathrm{GeV}$.

trino flux. Below $20 \mathrm{GeV}$, however, the atmospheric $\nu_{\tau}$ flux predominantly comes from the $\nu_{\mu}$ oscillations, i.e., $\phi_{\nu_{\tau}}^{\mathrm{tot}}(E) \approx \phi_{\nu_{\mu}}(E) \cdot \sin ^{2} 2 \theta \cdot \sin ^{2}\left(L / L_{\mathrm{osc}}\right)$ following Eq. (2). Since $L_{\mathrm{osc}} \equiv 4 E / \delta m^{2} \approx 330 \mathrm{~km}$ for $E=1 \mathrm{GeV}$ with $\delta m^{2}=2.4 \cdot 10^{-3} \mathrm{eV}^{2}$, we approximate $\sin ^{2}\left(L / L_{\mathrm{osc}}\right)$ with $\left(L / L_{\mathrm{osc}}\right)^{2}$ so that $\phi_{\nu_{\tau}}^{\text {tot }}(E) \sim \phi_{\nu_{\mu}}(E) E^{-2}$. Because the neutrino oscillation effect steepens the $\phi_{\nu_{\tau}}$ spectrum for $E \leq 20 \mathrm{GeV}$, the slope change of $\phi_{\nu_{\tau}}$ at $E \approx 20 \mathrm{GeV}$ is significant.

We have also worked out the comparison of the galactic-plane and the atmospheric $\nu_{\tau}$ fluxes for several other zenith angles. For instance, the crossing of the galactic-plane and the atmospheric $\nu_{\tau}$ fluxes occurs at $E=6.0 \mathrm{GeV}$ for the zenith angle $\xi=60^{\circ}$ for $\delta m^{2}=2.4 \cdot 10^{-3} \mathrm{eV}^{2}$ with the maximal mixing. Essentially, the atmospheric $\nu_{\tau}$ flux increases with the zenith angle $\xi$ while the galactic-plane $\nu_{\tau}$ flux stays unchanged. Moreover, from the phenomenological point of view, a larger $\xi$ only implies a larger atmospheric tau neutrino background while our focus is on the prospective observation of the astrophysical tau neutrinos.

Two key factors determine the angular behavior of the former flux. First, the atmosphere depth is larger for $\xi=$ $60^{\circ}$ compared to the downward direction. Second, the atmospheric muon neutrinos are produced more far away from the ground detector in this angle, implying a larger $\nu_{\mu} \rightarrow \nu_{\tau}$ oscillation probability. We have found that, for $E=10 \mathrm{GeV}$, the downward going muon neutrinos are produced on average about $13 \mathrm{~km}$ from the ground detector. At $\xi=60^{\circ}$, the above distance increases to 34 


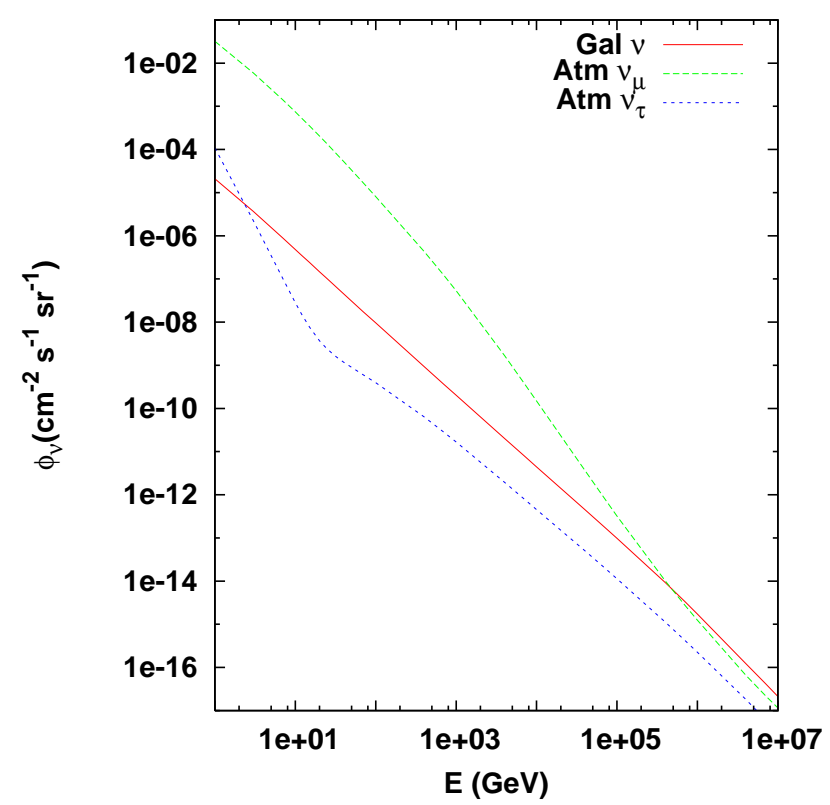

FIG. 6: An illustrative comparison of the downward going atmospheric $\nu_{\mu}$ and $\nu_{\tau}$ fluxes and the corresponding galacticplane neutrino fluxes in the presence of neutrino oscillations as a function of the neutrino energy in $\mathrm{GeV}$. The best fit values of the neutrino mixing parameters, namely, $\delta m^{2}=2.4 \cdot 10^{-3}$ $\mathrm{eV}^{2}$ and $\sin ^{2} 2 \theta=1$, are used. The galactic-plane and the atmospheric $\nu_{\mu}$ fluxes cross at $E=5 \cdot 10^{5} \mathrm{GeV}$.

$\mathrm{km}$. In the angular range $\xi<70^{\circ}$, the curvature of the Earth can be neglected and the angular dependence of the neutrino flux can be easily calculated [19].

In Fig. [5 we show the comparison for the zenith angle $\xi=180^{\circ}$ (vertically upward going direction). For $\delta m^{2}=2.4 \cdot 10^{-3} \mathrm{eV}^{2}$ with the maximal mixing the crossing between the two fluxes cross at $800 \mathrm{GeV}$. The oscillatory behavior in the total atmospheric tau neutrino flux is a manifestation of the narrow zenith angle dependence.

We have so far neglected the 3-body muon decay contribution to the atmospheric $\nu_{\mu}$ flux. As mentioned earlier, this contribution is relevant only for $1 \mathrm{GeV} \leq E \leq$ $10 \mathrm{GeV}$. The ratio of this contribution to the total atmospheric $\nu_{\mu}$ flux is available in the literature 16, 19]. For $\cos \xi=0.4\left(\xi=66^{\circ}\right), E=1 \mathrm{GeV}$, the ratio is about $50 \%$. It drops to $30 \%$ at $E=10 \mathrm{GeV}$ for the same $\xi[16$. This ratio is not very sensitive to the zenith angle $\xi$. The error due to neglecting this contribution propagates to the determination of the atmospheric $\nu_{\tau}$ flux by the neutrino oscillation effect. Numerically, this error is comparable to the uncertainty of the $\nu_{\tau}$ flux due to the uncertainty of the $\delta m^{2}$, as $\phi_{\nu_{\tau}}^{\text {tot }}(E) \approx \phi_{\nu_{\mu}}(E) \cdot \sin ^{2} 2 \theta \cdot\left(\delta m^{2} \cdot L / 4 E\right)^{2}$.

\section{CONCLUSIONS}

The results presented in Figs. 4 [5 and [ indicate the opportunities for the tau neutrino astronomy in the $\mathrm{GeV}$ energies for the incident zenith angles $0^{\circ} \leq \xi \leq 180^{\circ}$ in the two neutrino flavor mixing approximation. The galactic-plane tau neutrino flux dominates over the atmospheric tau neutrino background until a few GeV's. Furthermore, for $E \leq 20 \mathrm{GeV}$, the former flux has a rather different slope from that of the latter. This is an important criterion for distinguishing the two fluxes, particularly given that the normalization of the galacticplane tau neutrino flux is still uncertain.

We have pointed out that the dominance of the galactic-plane tau neutrino flux over its atmospheric background in $\mathrm{GeV}$ energies is unique among all the considered neutrino flavors. This is depicted in Fig. 6] Because of the $\nu_{\mu} \rightarrow \nu_{\tau}$ neutrino oscillations, the total galactic $\nu_{\tau}$ flux is identical to that of the galactic $\nu_{\mu}$ flux. However, the atmospheric $\nu_{\mu}$ flux is much larger than the atmospheric $\nu_{\tau}$ flux. As a result, in the presence of neutrino oscillations, the crossing energy value for the galactic-plane and the atmospheric $\nu_{\mu}$ fluxes is pushed up to $5 \cdot 10^{5} \mathrm{GeV}$, which is drastically different from the tau neutrino case.

We have estimated the galactic-plane tau neutrino induced shower event rate $N_{\nu_{\tau}}$ for the forthcoming one Mega ton class of detectors. It is obtained by convolving the total galactic-plane tau neutrino flux shown in Fig. [ 6 with the charged-current $\nu_{\tau} N$ interaction cross section (using the CTEQ6 parton distribution functions 20]). It is found that the $N_{\nu_{\tau}}$ can be 4.5 (7.5) for $E>10 \mathrm{GeV}$ with a data taking period of 3 (5) years.

A remark concerning the possible background induced by the electron neutrino events and the neutral current events to the prospective observation of tau neutrino events is in order. Below $10^{3} \mathrm{GeV}$, the tau lepton decay length is less than a mm. This tau lepton is produced in the detector in the galactic-plane tau neutrino induced interactions. There are certain specific signatures of the tau neutrino induced tau leptons such as the appearance of the kink at the tau lepton decay (absent for the electrons) 21], as well as the relative characteristic fractional energy sharing from the incident neutrino 22]. A large scale finely grained detector with a resolution of a few $\mu \mathrm{m}$ will thus be required to disentangle the galactic-plane tau neutrino induced events from the events induced by the electron neutrinos and the neutral current events on the event-by-event basis.

Acknowledgements.- H.A. thanks Physics Division of NCTS for support. F.F.L and G.L.L. are supported by the National Science Council of Taiwan under the grant number NSC92-2112-M-009-038. 
[1] Y. Ashie et al. [Super-Kamiokande Collaboration], Phys. Rev. Lett. 93, 101801 (2004). For a more recent and detailed discussion, see, ibid., arXiv:hep-ex/0501064

[2] Y. Suzuki, in Proceedings of the 28th International Cosmic Ray Conferences (ICRC 2003), Tsukuba, Japan, 2003, edited by T. Kajita et al. (Universal Academic Press, Inc., Tokyo, Japan, 2004), Vol. 8, p. 75.

[3] T. Kajita and Y. Totsuka, Rev. Mod. Phys. 73, 85 (2001). For a recent discussion of the forthcoming astrophysical neutrino telescopes, see, A. B. McDonald et al., Rev. Sci. Instrum. 75, 293 (2004).

[4] See, for instance, H. Athar, Chin. J. Phys. 42, 1 (2004).

[5] H. Athar, Mod. Phys. Lett. A 19, 1171 (2004).

[6] T. K. Gaisser and M. Honda, Ann. Rev. Nucl. Part. Sci. 52, 153 (2002).

[7] G. Ingelman and M. Thunman, arXiv:hep-ph/9604286

[8] T. K. Gaisser, Astropart. Phys. 16, 285 (2002).

[9] T. K. Gaisser, Cosmic Rays And Particle Physics (Cambridge University Press, Cambridge, UK, 1990).

[10] T. Sjöstrand, Comput. Phys. Commun. 82, 74 (1994).

[11] H. Athar, K. Cheung, G. L. Lin and J. J. Tseng, As- tropart. Phys. 18, 581 (2003).

[12] L. Pasquali, M. H. Reno and I. Sarcevic, Phys. Rev. D 59, 034020 (1999).

[13] P. Nason, S. Dawson and R. K. Ellis, Nucl. Phys. B 327, 49 (1989) [Erratum-ibid. B 335, 260 (1990)].

[14] M. L. Mangano, P. Nason and G. Ridolfi, Nucl. Phys. B 373, 295 (1992).

[15] E. V. Bugaev et al., Nuovo Cimento C 15, 401 (1992).

[16] P. Lipari, Astropart. Phys. 1, 195 (1993).

[17] L. Pasquali and M. H. Reno, Phys. Rev. D 59, 093003 (1999).

[18] B. A. Li, Phys. Rev. D 52, 5165 (1995).

[19] T. K. Gaisser and T. Stanev, Phys. Rev. D 57, 1977 (1998).

[20] S. Kretzer, H. L. Lai, F. I. Olness and W. K. Tung, Phys. Rev. D 69, 114005 (2004).

[21] See, for instance, H. Pessard [OPERA Collaboration], arXiv:hep-ex/0504033 and references cited therein.

[22] For details, see, T. Stanev, Phys. Rev. Lett. 83, 5427 (1999). 